\title{
Change in the Heterogeneous Distribution of Tubulin Isotypes in Mitotic Microtubules of the Sea Urchin Egg by Treatment with Microtubule Depolymerizing or Stabilizing Drugs.
}

\author{
Mikako T. Oka ${ }^{1}$, Takao Arai ${ }^{2}$, and Yukihisa Hamaguchi ${ }^{1 *}$ \\ ${ }^{1}$ Biological Laboratory, Faculty of Science, Tokyo Institute of Technology, Tokyo 152 and ${ }^{2}$ Department of Applied \\ Biological Science, Faculty of Science and Technology, Science University of Tokyo, Noda, Chiba 278, Japan
}

Key words: sea urchin egg/microtubule heterogeneity/mitotic apparatus/tubulin isotypes/hexylene glycol/colcemid

\begin{abstract}
$A B S T R A C T$. In the mitotic sea urchin egg, the spindle microtubules were composed of different tubulin isotypes from those of astral microtubules using monoclonal antibodies [Oka et al. (1990) Cell Motil. Cytoskeleton, 16, 239-250]. Three of the antibodies, D2D6, DM1B, and YL1/2, were specific for spindle microtubules, astral microtubules and reactive with both microtubules, respectively. The mitotic sea urchin egg was treated with microtubule depolymerizing (colcemid and nocodazole) and stabilizing (hexylene glycol) drugs and change in the heterogeneous distribution of the tubulin isotypes was investigated by the immunofluorescence procedure using these three monoclonal anti-tubulin antibodies. We observed that: (1) the microtubule depolymerizing drugs caused quick depolymerization of most mitotic microtubules, and a small number of spindle microtubules remaining were stained with all three antibodies; (2) hexylene glycol induced many microtubules in the mitotic apparatus, which was stained with D2D6 but was not stained with DM1B; (3) hexylene glycol also induced a great number of miniasters in the cytoplasm, and they were stained with three antibodies. These results suggest that these drugs altered the distribution of tubulin isotypes in the mitotic microtubules during depolymerization or polymerization within a short time.
\end{abstract}

During cell division of echinoderm eggs, the spindle and asters of the mitotic apparatus play their own roles in both karyokinesis and cytokinesis as follows. The spindle forces chromosomes to align in the equator during prometaphase and to separate into two sister chromatid groups toward the spindle poles during anaphase (anaphase A), whereas the asters pull the poles apart during anaphase (anaphase B) $(14,16)$ and establish a furrow to the equatorial cortex to divide the cytoplasm into two daughter cells (22). The spindle and asters are mainly composed of microtubules. Therefore, it is important to determine the relationship between the structure and function of microtubules in dividing cells. Recently we reported that some tubulin isotypes of the spindle are different from those of the aster (19). However, the biological significance of the heterogeneous distribution of tubulin isotypes is not yet clear in the mitotic cell. On the other hand, it is known that in the interphase cell, stability of microtubules was dependent on their tubulin isotypes $(17,20)$ and that functionally differentiated microtubules were composed of different isotypes $(1,2)$.

\footnotetext{
* To whom correspondence and reprint requests should be addressed.
}

It is well known that some mitotic poisons affect the polymerization of tubulin and the stability of microtubules, which results in inhibition of mitosis. Colcemid and nocodazole depolymerize microtubules and hexylene glycol stabilizes microtubules. Using these drugs, characteristics of microtubules such as microtubule dynamics and microtubule organizing activity have been investigated, especially in living cells (for a review, see ref. 5). Rapid depolymerization and polymerization of mitotic microtubules were also shown in the egg treated with microtubule depolymerizing and stabilizing drugs $(6,7,11,13,23,26,27)$. In order to study the controlling mechanism of heterogeneous distribution of tubulin isotype in microtubules and the relationship between the distribution and microtubule function in the mitotic apparatus, sea urchin eggs were treated with those mitotic poisons and the heterogeneous distribution of tubulin isotypes was examined by indirect immunofluorescence. For detecting the heterogeneity, we used three kinds of monoclonal anti-tubulin antibodies which stained the mitotic apparatus in three different patterns, as described previously (19). In this study, we report that the heterogeneous patterns of the mitotic apparatus stained with the anti-tubulin antibodies, as well as its morphology, were altered by the treatment with the mi- 
totic poisons.

\section{MATERIALS AND METHODS}

Drugs. Colcemid dissolved at $1 \mathrm{mM}$ in deionized water, and nocodazole dissolved at $20 \mathrm{mM}$ in dimethylsulfoxide were stored at $-20^{\circ} \mathrm{C}$, and diluted with Ca-free artificial sea water (Jamarin Laboratory, Osaka) just prior to use. Hexylene glycol was dissolved at $10 \%$ or $20 \%$ in Ca-free sea water, stored, and used after diluting with $\mathrm{Ca}$-free sea water just prior to use $(6,23)$.

Treatment with microtubule poisons and observation by indirect immunofluorescence. The gametes of the sea urchin, Hemicentrotus pulcherrimus, and the sand dollar, Clypeaster japonicus, were obtained and the zygotes were allowed to develop as described previously (19). The suspension of developing eggs gathered at the bottom of a dish was mixed with more than a 50 -fold greater volume of 30 or $60 \mu \mathrm{M}$ colcemid, 4 or $40 \mu \mathrm{M}$ nocodazole, or $5,7.5,10$, or $20 \%$ hexylene glycol in $\mathrm{Ca}$-free sea water using a glass capillary. After an appropriate time, these treated eggs were put into an extract buffer $(10$ mM EGTA, $0.55 \mathrm{mM} \mathrm{MgCl}_{2}, 25 \%$ glycerol, $1 \%$ Nonidet P-40, $2.5 \mathrm{mM}$ phenylmethylsulfonyl fluoride, and $25 \mathrm{mM} \mathrm{MES}, \mathrm{pH}$ 6.8). For premitotic treatment, eggs before insemination or 25 min after insemination were treated with $2 \mu \mathrm{M}$ colcemid for 2.5 or $4.5 \mathrm{~min}$ and allowed to develop after washing 3 or 4 times with Ca-free sea water. The extraction and indirect immunofluorescence were performed as described previously (19). The first antibodies used in this study were D2D6, DM1B, and YL1/2, which are specific for spindle microtubules, astral microtubules, and reactive with both microtubules, respectively, as described previously (19). YL1/2, a monoclonal anti-tubulin antibody specific to tyrosinated $\alpha$ tubulin (18), was purchased from Sera-Lab, England. DM1B, a monoclonal anti- $\beta$-tubulin antibody (3), was purchased from Amersham, England. D2D6, a monoclonal anti-tubulin antibody against squid nerve $\alpha$-tubulin was raised by Arai and Matsumoto (1).

Observation was carried out by fluorescence and differential interference contrast microscopy, and photographs were taken on $35 \mathrm{~mm}$ film as described previously (19).

\section{RESULTS}

For this study, we used two species of sea urchins, Clypeaster japonicus and Hemicentrotus pulcherrimus, and obtained essentially the same results. Therefore, only the results obtained from $H$. pulcherrimus are shown in this paper.

\section{Effect of microtubule depolymerizing drugs}

a. Colcemid. The mitotic sea urchin egg was treated with $60 \mu \mathrm{M}$ colcemid and the change in the reactivity of the mitotic apparatus with three monoclonal anti-tubulin antibodies, YL1/2, D2D6, and DM1B, was observed. The spindle of the nontreated egg was stained with YL1/2 and D2D6 but not with DM1B (Figs. 1a, b, c), as previously reported (19). After $2 \mathrm{~min}$ of the treatment, most of the astral microtubules were depolymerized, and the spindle slightly diminished in size (Fig. 1d). The diminished spindle became stained with DM1B and with the other two antibodies, YL1/2 and D2D6, as did in nontreated cells (Figs. 1d, e, f). After $5 \mathrm{~min}$, the astral microtubules depolymerized completely and the spindle shrank much more than the spindle of the 2min-treated egg. The shrinking spindle was also stained with the three antibodies (Figs. 1g, h, i). These results indicated that the heterogeneous distribution of tubulin isotypes in the spindle microtubules was changed by the $60 \mu \mathrm{M}$-colcemid treatment.

Next, the mitotic eggs were treated with colcemid at the lower $(30 \mu \mathrm{M})$ concentration. After 2 min treatment, the mitotic apparatus was stained with each antibody in patterns similar to normal mitotic apparatus, although the mitotic apparatus became a little smaller. When treatment was prolonged to $4 \mathrm{~min}$, few microtubules in the aster remained around the poles, and the spindle shrank. The staining pattern of the mitotic apparatus with D2D6 was naturally the same as that of YL1/2, because few astral microtubules remained. However, the spindle became stained with DM1B as with the case of $60 \mu \mathrm{M}$ (data not shown).

Premitotic treatment with colcemid at low concentration (1-2 $\mu \mathrm{M})$ reduces the tubulin pool which can assemble to microtubules in the egg cytoplasm. As a result of the small size of the tubulin pool, a small mitotic apparatus formed in the egg, although the egg completed division according to Sluder (29). We treated the premitotic egg with $2 \mu \mathrm{M}$ colcemid for 2.5 or 4.5 minutes and observed the reactivity of the small mitotic apparatus in colcemid-treated egg with the antibodies. The staining patterns of the small mitotic apparatus were the same as those in normal cells, as shown in Fig. 2. D2D6 stained the spindle strongly but the asters faintly (Figs. 2b, e), and DM1B stained only the asters (Figs. 2c, f). It was confirmed that these treated eggs completed division. These results indicated that the patterns of functionally normal mitotic apparatus stained with DM1B and D2D6 are exclusive whether its size is normal or small.

b. Nocodazole. Nocodazole depolymerizes microtubules more rapidly than colcemid. The eggs were treated with a high concentration $(40 \mu \mathrm{M})$ of nocodazole, and the rapid change in the distribution of tubulin isotypes was observed. Staining with YL1/2 showed that the asters and spindle became small after $20 \mathrm{sec}$ of treatment (Figs. 3a, d). At this time, D2D6 and DM1B stained specifically the spindle and the asters, respectively (Figs. 3e, f), similar to the case of nontreated eggs (Figs. $3 \mathrm{~b}, \mathrm{c}$ ). In the case of $40 \mathrm{sec}$ treatment, most of the astral microtubules depolymerized, but spindle microtubules still remained, although the spindle became small. 


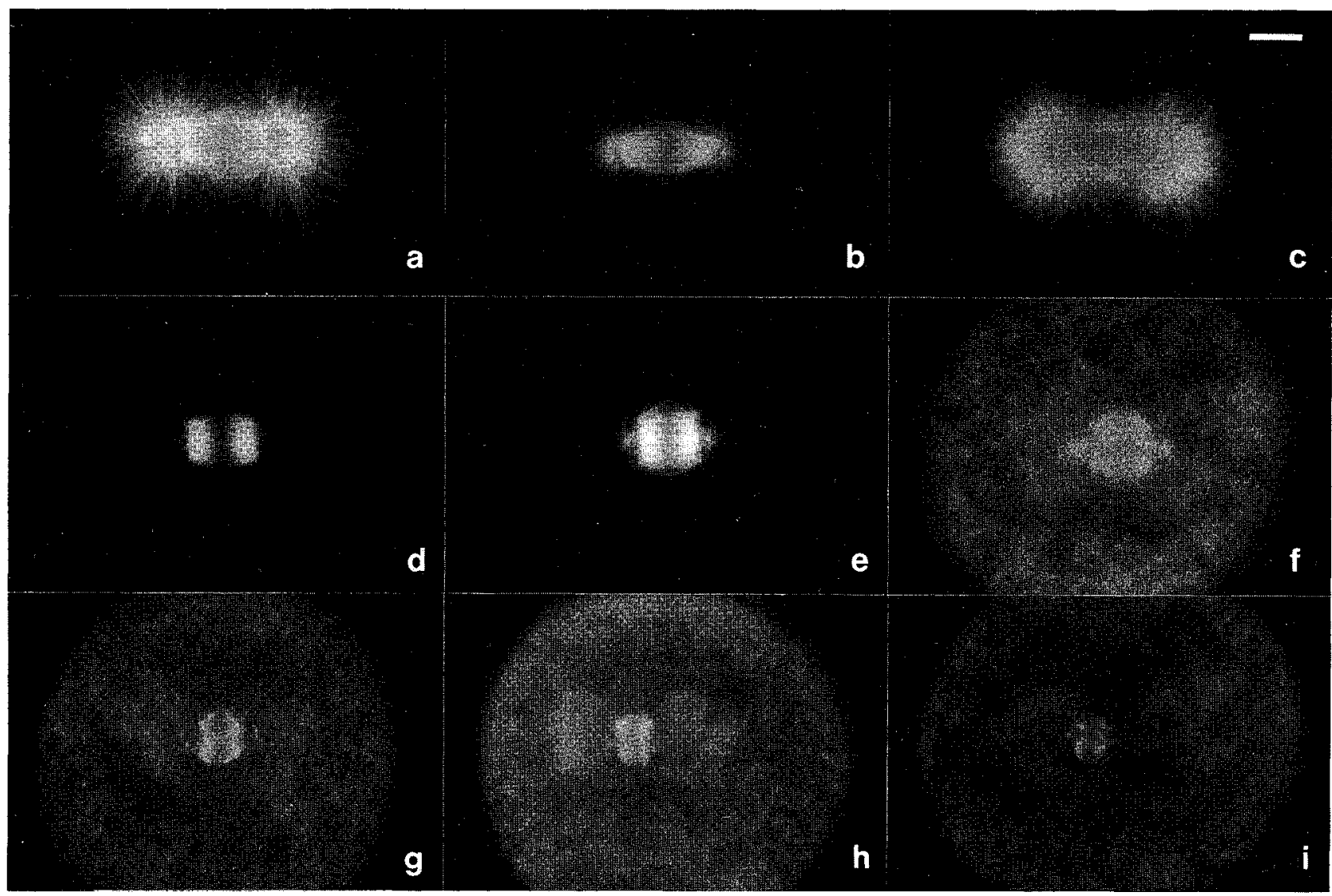

Fig. 1. Metaphase mitotic apparatuses treated with $60 \mu \mathrm{M}$ colcemid. a, b, and c; nontreated (control) eggs. d, e, and f; eggs treated for 2 min. g, h, and i; eggs treated for 5 min. a, d, and g; eggs stained with YL1/2. b, e, and h; eggs stained with D2D6. c, f, and i; eggs stained with DM1B. The bar shows $10 \mu \mathrm{m}$.

These microtubules in the small spindle became reactive with DM1B as well as with D2D6 and YL1/2 (Figs. 3g, $h, \mathrm{i})$. All microtubules in the egg depolymerized with 3 min treatment (data not shown).

Figure 4 shows fluorescence micrographs of the eggs treated with nocodazole at the lower concentration (4 $\mu \mathrm{M})$. By 1 min treatment, a considerable fraction of the astral microtubules depolymerized and the spindle became short and narrow (Figs. 4a, b, c). These spindles became stained slightly with DM1B (Fig. 4c); however, heterogeneous staining patterns obtained by D2D6 and DM1B were not changed completely. By 3 min treatment, most of the astral microtubules depolymerized and spindle microtubules shrank further and became bundles, which is a different phenomenon from that in the egg treated with $40 \mu \mathrm{M}$ nocodazole and with colcemid. It seems likely that the main constituent of the bundle is kinetochore microtubules. The bundle was stained with DM1B (Fig. 4f), indicating that the difference in patterns stained with antibodies disappeared, as shown in the eggs treated with nocodazole at higher con- centration.

Effect of hexylene glycol. Hexylene glycol, which is known as a microtubule-stabilizing drug, induced microtubule polymerization in mitotic sea urchin eggs, and resulted in the increase in the number of spindle and astral microtubules, as reported by Rebhun and Sawada (23), and Endo et al. (6). In the case of 5\% hexylene glycol treatment, spindle microtubules polymerized and reached the equator during all mitotic stages (Fig. 5), but chromosomes remained at the same position in the spindle as before the treatment. This indicates that the mitotic stage of each egg at the time when hexylene glycol treatment started can be judged from the position of the chromosomes. The mitotic apparatus became slightly smaller, although microtubules were polymerized and increased in number and changed to the form of two concentrated spheres. Therefore, the mitotic apparatus was not only stabilized, but its morphology was also unusually transformed by the treatment with the microtubule-stabilizing drug, hexylene glycol. D2D6 became reactive with the amphiaster as well as 


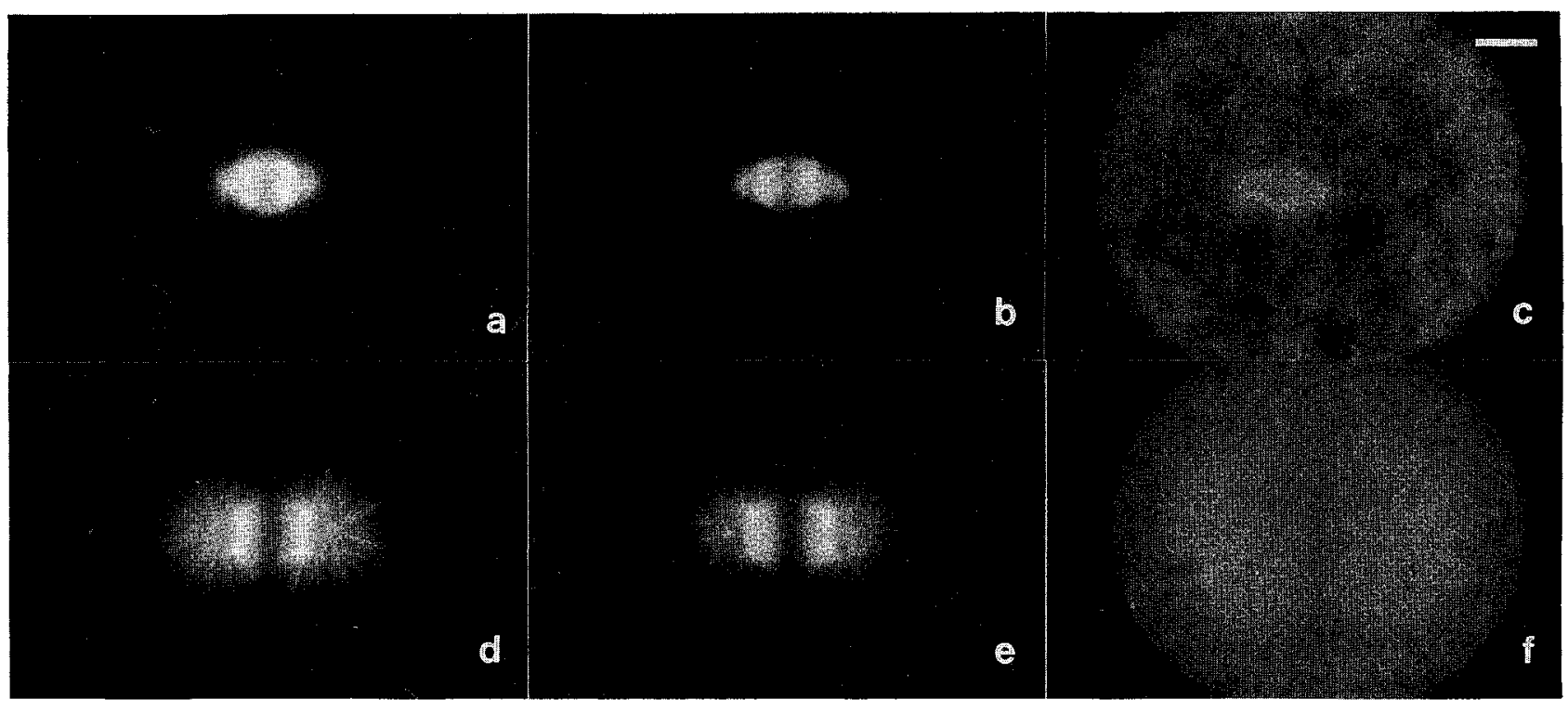

Fig. 2. Mitotic apparatuses treated for 4 min with $2 \mu \mathrm{M}$ colcemid 25 min after fertilization. a, b, and c; metaphase. d, e, and f; anaphase. a and $\mathrm{d}$; eggs stained with YL1/2. b and e; eggs stained with D2D6. $\mathrm{c}$ and f; eggs stained with DM1B. It is noted that staining patterns are the same as in control eggs. The bar shows $10 \mu \mathrm{m}$.

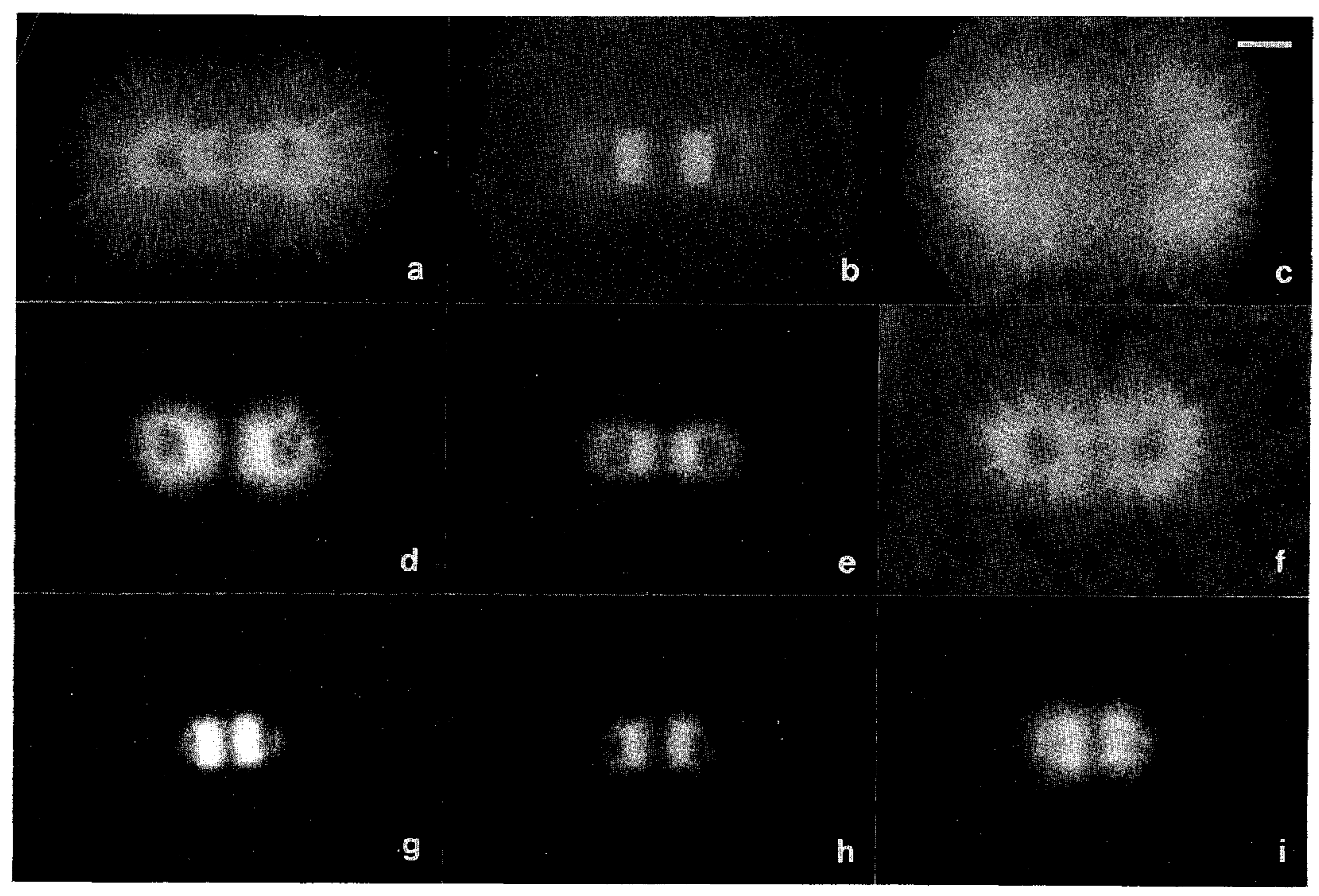

Fig. 3. Anaphase mitotic apparatuses treated with $40 \mu \mathrm{M}$ nocodazole. a, b, and c; nontreated (control) eggs. d, e, and f; eggs treated for 20 sec. $\mathrm{g}, \mathrm{h}$, and i; eggs treated for 40 sec. a, d, and g; eggs stained with YL1/2. b, e, and h; eggs stained with D2D6. c, f, and i; eggs stained with DM1B. The bar shows $10 \mu \mathrm{m}$. 


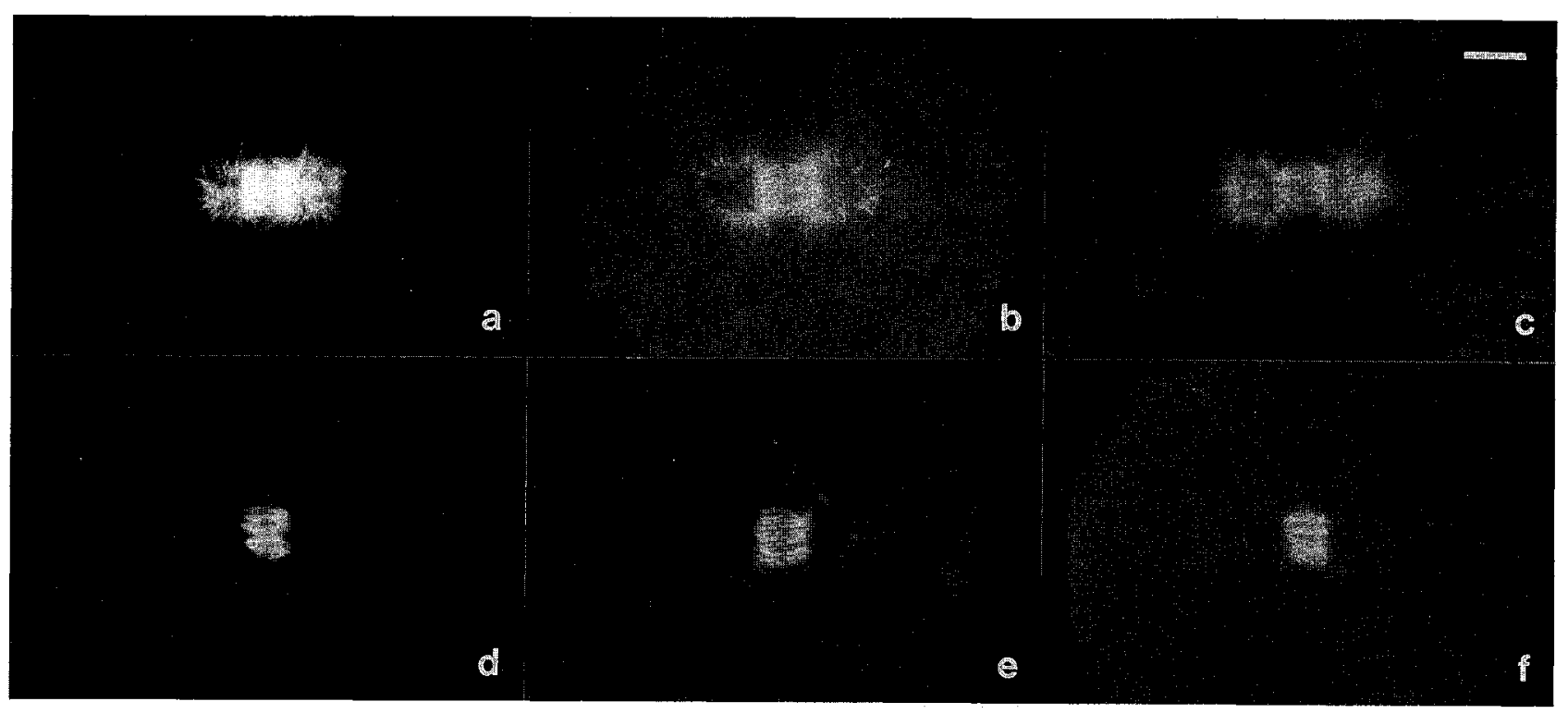

Fig. 4. Metaphase mitotic apparatuses treated with $4 \mu \mathrm{M}$ nocodazole. $\mathrm{a}, \mathrm{b}$, and $\mathrm{c}$; eggs treated for $1 \mathrm{~min}$. $\mathrm{d}$, e, and f; eggs treated for $3 \mathrm{~min}$. a and d eggs stained with YL1/2. b and e, were stained with D2D6. $\mathrm{c}$ and $\mathrm{f}$ were stained with DM1B. In 3-min-treated egg, spindle (kinetochore) microtubules were bundled and became stained with DM1B as well as with YL1/2 and D2D6. The bar shows $10 \mu \mathrm{m}$.

with the spindle, which indicates that D2D6 became reactive with whole microtubules in the egg instead of YL1/2 (Figs. $5 b, e, h$ ) because the central region of the mitotic apparatus lost its reactivity with YL1/2 (Figs. $5 \mathrm{a}, \mathrm{d}, \mathrm{g})$. DM1B did not stain or only slightly stained the mitotic apparatus (Figs. 5c, f, i). However, DM1B stained the peripheral regions of the asters in later stages (anaphase and telophase). In this experiment, microtubule stabilization caused by the hexylene glycol changed not only the morphology of the mitotic apparatus, but also the distribution of tubulin isotypes; astral microtubules became reactive with D2D6 and lost reactivity with DM1B.

In the peripheral cytoplasm of the metaphase egg treated with 5\% hexylene glycol, many cytasterlike structures, which were called "miniasters" by Endo et al. (7), appeared. The number of miniasters in an egg decreased but microtubules in single miniasters increased when the treatment time increased (Figs. 5d, g). At anaphase, instead of miniasters, network-like microtubules without centers polymerized in the peripheral cytoplasm. These patterns of cytoplasmic microtubules such as miniasters and network-like microtubules agreed with those reported by Endo et al. (7). All three antibodies stained peripheral miniasters and network-like microtubules brightly (Figs. $5 \mathrm{~g}, \mathrm{~h}, \mathrm{i}$ ), which indicates that there is no heterogeneity in newly polymerized microtubules in the egg cytoplasm.

In order to stabilize microtubules more quickly, eggs were treated with hexylene glycol at higher concentrations $(7.5,10$, or $20 \%)$ for short times. The higher the concentration of hexylene glycol, the more and the shorter microtubules polymerized in the cytoplasm within 2 min (Fig. 6). At metaphase, when the treatment with $7.5 \%$ or $10 \%$ hexylene glycol was prolonged to 10 min, miniasters appeared in the peripheral cytoplasm and each miniaster in the egg treated with $10 \%$ hexylene glycol had fewer microtubules than in that treated with $7.5 \%$ hexylene glycol. However, miniasters did not form at 20\% hexylene glycol, whereas many short microtubules polymerized randomly. These polymerized microtubules were stained with all three antibodies (Figs. 6g, h, i). On the other hand, the number of microtubules polymerizing in the mitotic apparatus decreased when the concentration of hexylene glycol increased to $10 \%$ (Figs. 6d, e, f). Increase in the microtubule number did not occur in the mitotic apparatus, or the mitotic apparatus did not transform at 20\% (Figs. $6 \mathrm{~g}, \mathrm{~h}, \mathrm{i}$ ). Therefore, the mitotic apparatus in these micrographs appears to decrease in contrast to observation by fluorescence microscopy because of the increase in fluorescence intensity of immunofluorescently stained cytoplasmic microtubules, but not because of the decrease in the fluorescence intensity of the mitotic apparatus. These phenomena seem to occur because all unpolymerized tubulin in the egg cytoplasm before treatment polymerized at once in the cytoplasm at a high concentration of hexylene glycol, independent of the neighboring microtubule nucleating center or the end of microtubules. 


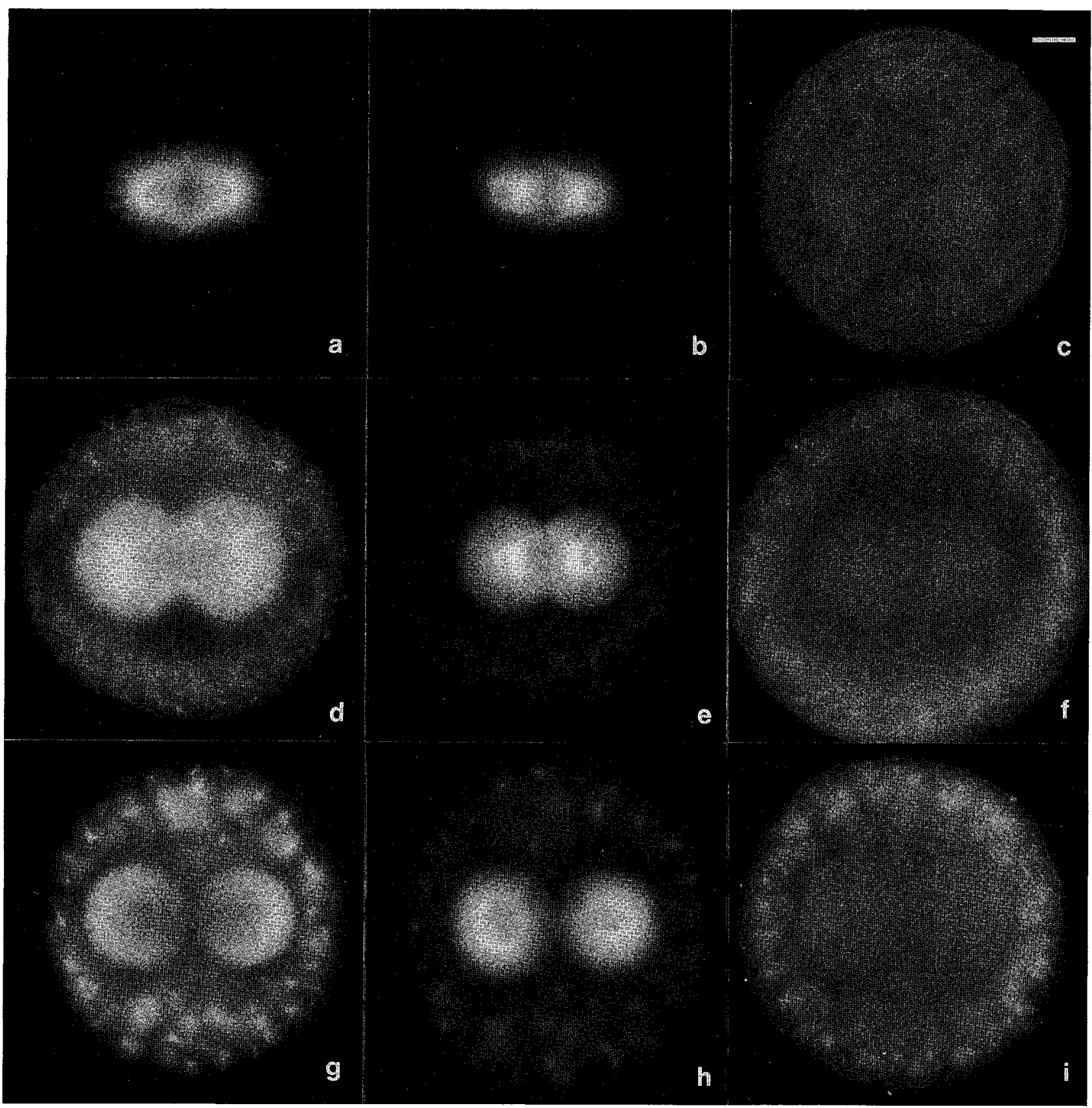

Fig. 5. Metaphase mitotic apparatuses treated with $5 \%$ hexylene glycol. a, b, and c; eggs treated with hexylene glycol for 30 sec. d, e, and f; eggs treated for $5 \mathrm{~min}$. g, h, and i; eggs treated for $30 \mathrm{~min}$. a, d, and g; eggs stained with YL1/2. b, e, and h; eggs stained with D2D6. c, f, and i; eggs stained with DM1B. DM1B stained many miniasters strongly but the mitotic apparatus slightly. The bar shows $10 \mu \mathrm{m}$.

\section{DISCUSSION}

Morphological changes of the mitotic apparatus in the sea urchin egg by treatment with microtubule poisons. Spindle microtubules of the sea urchin or sand dollar egg were more stable than astral microtubules to the treatment with microtubule depolymerizing drugs, colcemid and nocodazole. Furthermore, the spindle microtubules became bundles by treatment with a low concentration of nocodazole. In general, kinetochore microtubules are more stable than non-kinetochore microtubules. It was reported that in the mitotic apparatus of a PtK1 cell treated with nocodazole, kinetochore 


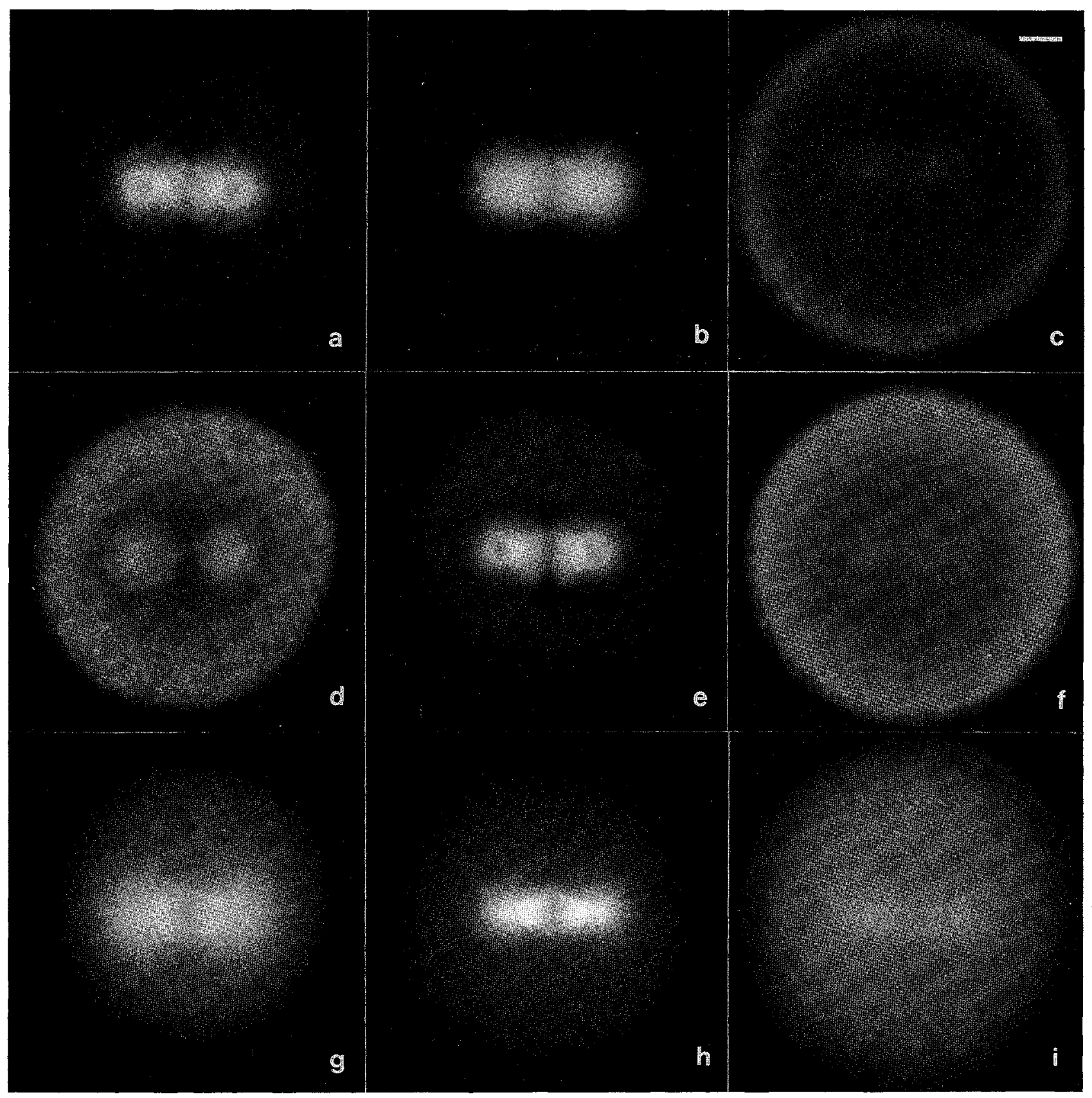

Fig. 6. Metaphase mitotic apparatuses treated with the higher concentrations of hexylene glycol. a, b, and c; eggs treated with $7.5 \%$ hexylene glycol. d, e, and f; eggs treated with 10\% hexylene glycol. g, h, and i; eggs treated with $20 \%$ hexylene glycol. All eggs were treated for 2 min. a, d, and g; eggs stained with YL1/2. b, e, and h; eggs stained with D2D6. c, f, and i; eggs stained with DM1B. Short microtubules polymerized in the cytoplasm were stained with all antibodies. Few microtubules polymerized in the mitotic apparatus treated with $20 \%$ hexylene glycol. The bar shows $10 \mu \mathrm{m}$.

microtubules were more stable than non-kinetochore microtubules $(30,31)$. The former microtubules were also more stable than the latter to cold treatment in PtK1 cells and crane fly spermatocytes $(24,25,28)$. Therefore, the bundles of spindle microtubules were considered to be that of kinetochore microtubules. When spindle microtubules were treated with microtubule-depolymerizing drugs for $20 \mathrm{sec}$ to a few min, the spindle became short. Inoué (15) observed that the chromosomes moved to the egg surface by colchicine treat- 
ment when the spindle of Chaetopterus oocyte shrank. These facts indicate the presence of interactions among the spindle microtubules during depolymerization. Therefore, the bundles of spindle microtubules in the egg treated with $4 \mu \mathrm{M}$ nocodazole are thought to be formed as a result of interaction among the kinetochore microtubules. The bundling of spindle microtubules was also reported in the PtK1 cell treated with nocodazole $(30,31)$ and in the PtK1 cell (24) and crane fly cells $(25,28)$ treated at low temperature.

Relationship between distinct function and different distribution of tubulin isotypes in the mitotic microtubules of the sea urchin. We have recently shown that there is a difference in tubulin isotypes between spindle and astral microtubules in the mitotic apparatus of the sea urchin eggs using monoclonal anti-tubulin antibodies (19). In this study, we demonstrate that the treatment of the mitotic eggs with microtubule poisons caused the transformation of the mitotic apparatus and changes in the heterogeneous distribution of tubulin isotypes in the mitotic apparatus. It should be noted that the treatment caused a loss of the function of the mitotic apparatus. The premitotic treatment with colcemid for a short period made the mitotic apparatus small. However, the treatment caused neither loss of the function of the mitotic apparatus nor changes in the heterogeneous distribution of tubulin isotypes. These results suggest that the loss of the function is due to the change in the distribution of the tubulin isotypes. This conclusion strongly supports the multi-tubulin hypothesis that microtubules composed of different isotypes are in- volved in different cellular functions $(4,8,21)$.

The mechanism of heterogeneous distribution of tubulin isotypes in the mitotic apparatus and the change in the distribution by the treatment with the mitotic poisons. Distributions of tubulin isotypes in the sea urchin mitotic apparatus are summarized schematically in Fig. 7. The rows of A, B, and C in Fig. 7 show the mitotic microtubular structures at depolymerizing, normal, and stabilizing states, respectively. The columns in the figure show microtubule reactivity with three monoclonal antibodies, D2D6, YL1/2, and DM1B. In normal eggs, D2D6 stains the spindle and the center of asters (Fig. 7d), YL1/2 stains the whole mitotic apparatus (Fig. 7e), and DM1B stains only the asters (Fig. 7f). As described previously, it is reported that the spindle microtubules are more stable than the astral microtubules in the sea urchin. These results strongly suggest that the stable microtubules are composed of different tubulin isotypes from those of the unstable microtubules. D2D6 and DM1B react specifically with the isotypes localized in the stable and the unstable microtubules, respectively, and YL1/2 reacts with the isotypes distributed in both microtubules. D2D6 also stains the spindle even after the asters depolymerized (Fig. 7a). In the stabilizing state by the treatment with hexylene gly$\mathrm{col}$, astral microtubules become stable and therefore obtain the reactivity with D2D6 (Fig. 7g). DM1B stains the periphery of the amphiaster and miniasters in the peripheral cytoplasm in the stabilizing state (Fig. 7i). In the depolymerizing state after the asters disappear, the spindle microtubules became unstable and changed to be

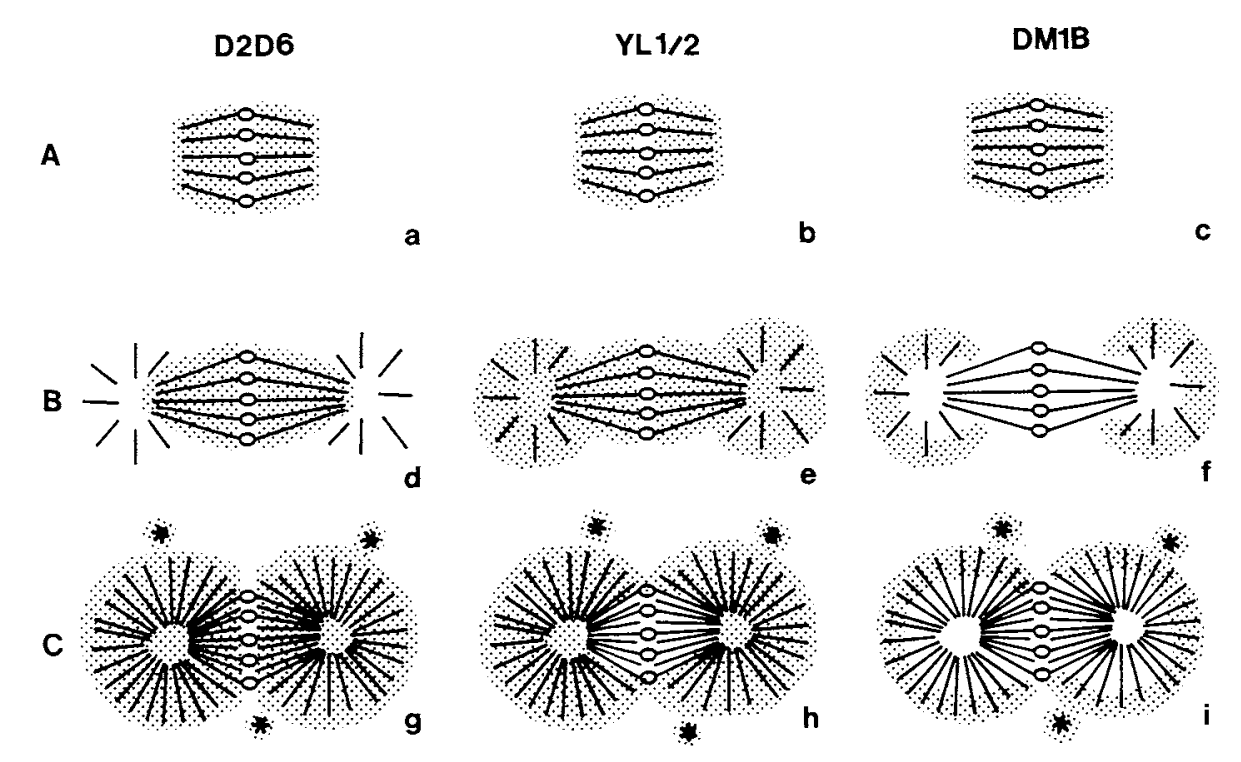

Fig. 7. A scheme indicating the microtubule stability and the staining pattern of the mitotic apparatus. a, d, and g; staining patterns with D2D6. b, e, and h; staining patterns with YL1/2. c, f, and i; staining patterns with DM1B. (A) the mitotic apparatus in depolymerizing state, (B) the normal mitotic apparatus, (C) the mitotic apparatus in stabilizing state. Dotted areas show immunofluorescently stained regions of microtubular structures. 
stained with DM1B (Fig. 7c). The weak reactivity of spindle microtubules with YL1/2 in the stabilizing state (Fig. 7h) may be caused by the detyrosination of $\alpha$-tubulin in the microtubules after polymerization. It has been reported that tyrosinated $\alpha$-tubulin converted to detyrosinated tubulin after polymerization (10) and the detyrosinated tubulin was distributed in the spindle but not in the aster (9).

The heterogeneous distribution of tubulin isotypes in the spindle and the aster is easily realized when enough tubulin is present in the cytoplasm. In other words, a tubulin isotype is favored to polymerize to microtubules composed of the same isotype in corporation to microtubules composed of different isotypes when the concentration of the nonpolymerized tubulin is high. In contrast, polymerization of some tubulin isotypes to microtubules composed of other isotypes frequently occurs when the tubulin pool is not large enough. Both microtubule-depolymerizing and -polymerizing drugs reduce the tubulin pool. The former binds to nonpolymerized tubulin and makes it unable to polymerize. The latter causes a decrease in the critical concentration for polymerization and reduces the concentration of nonpolymerized tubulin. Two mechanisms can be considered to explain the change in the heterogeneity of mitotic microtubules: (1) modification of mitotic microtubules after polymerization alters the isotype of tubulin in the mitotic apparatus, and (2) tubulin subunits of mitotic microtubules exchange with other tubulin isotypes in the cytoplasm by means of dynamic instability and/ or subunit exchange along the microtubule. However, we cannot completely rule out the possibility that the change in the distribution of microtubule-associated protein(s) alters the staining pattern because the microtubule-associated protein(s) might block the interaction between the monoclonal antibody and the tubulin isotype. It seems likely that (1) explains the hexylene glycol-induced change in the reactivity of spindle microtubules with YL1/2 as described previously (Fig. 7h). From (2), the following is expected. By reducing the pool by means of nocodazole or colcemid treatment, subunits in depolymerizing kinetochore microtubules would exchange with all isotypes in the cytoplasm. This random exchange results in the phenomenon where in the remaining kinetochore microtubules are reactive with all antibodies, because it is known that all tubulin molecules of microtubules in the sea urchin mitotic apparatus exchange with cytoplasmic tubulin within 30 sec $(12,26)$. The answer will be found when quantitative analysis of the tubulin isotypes, before and after treatment with microtubule-poisoning drugs, is carried out. If (1) is true, the amount of each tubulin isotype will change during the treatment, and if (2) is true, the amount of each tubulin isotype will not change.
Acknowledgments. We wish to thank Prof. Hikoichi Sakai of the University of Tokyo for his critical reading of the manuscript. We thank Dr. G. Matsumoto (Electrotechnical Lab.) and Shin-ichi Hisanaga (Tokyo Institute of Technology) for their valuable discussions. We thank also the staff of the Misaki Marine Biological Station for supplying the sand dollars.

This work was supported by Grants-in-Aid from the Ministry of Education, Science, and Culture of Japan to T.A. (01658001) and Y.H. (62540538).

\section{REFERENCES}

1. Arai, T. and Matsumoto, G. (1988). Subcellular localization of functionally differentiated microtubules in squid neurons.: Regional distribution of microtubule-associated proteins and $\beta$ tubulin isotypes. J. Neurochem., 51: 1825-1838.

2. Arai, T., Ichikawa, M., and Matsumoto, G. (1989). Functional differentiation of microtubules localized in the subaxolemmal axoplasm of the squid giant axon. In Dynamics of Microtubules: Proceeding of the 14th Taniguchi international symposium (H. Hotani, ed.). The Taniguchi Foundation, Kyoto, pp 260-282.

3. Blose, S.H., Meltzer, D.I., and Feramisco, J.R. (1984). 10$\mathrm{nm}$ filaments are induced to collapse in living cells microinjected with monoclonal and polyclonal antibodies against tubulin. $J$. Cell Biol., 98: 874-858.

4. Clevveland, D.W. and Sullivan, K.F. (1986). Molecular biology and genetics of tubulin. Annu. Rev. Biochem., 54: 331-365.

5. Dustin, P. (1984). Microtubules. 2nd ed. Springer-Verlag, Berlin, $482 \mathrm{pp}$.

6. Endo, S., Toriyama, M., and Sakai, H. (1983). The mitotic apparatus with unusually many microtubules from sea urchin eggs treated by hexyleneglycol. Dev. Growth Differ., 25: 307314.

7. Endo, S., Toriyama, M., Ohta, K., and SAKai, H. (1990). Formation of miniasters in the cytoplasm of hexyleneglycoltreated sea urchin eggs. Cell Motil. Cytoskeleton, 15: 23-33.

8. Fulton, C. and Simpson, P.A. (1976). Selective synthesis and utilization of flagellar tubulin. The multi-tubulin hypothesis. In Cell Motility (R. Goldman, T. Pollard, and J.L. Rosenbaum, eds.) Cold Spring Harbor Press, Cold Spring Harbor, New York, pp987-1005.

9. Gundersen, G.G. and Bulinski, J.C. (1986). Distribution of tyrosinated and nontyrosinated $\alpha$-tubulin during mitosis. J. Cell Biol., 102: 1118-1126.

10. Gundersen, G.G., Khawaja, S., and Bulinski, J.C. (1987). Postpolymerization detyrosination of $\alpha$-tubulin: A mechanism for subcellular differentiation of microtubules. J. Cell Biol., 105: 251-264.

11. HamaguCHI, Y. (1975). Microinjection of colchicine into sea urchin eggs. Dev. Growth Differ., 17: 111-117.

12. Hamaguchi, Y., Toriyama, M., Sakai, H., and Hiramoto, Y. (1985). Distribution of fluorescently labeled tubulin injected into sand dollar eggs from fertilization through cleavage. J. Cell Biol., 100: 1262-1272.

13. Hamaguchi, Y., Toriyama, M., Sakai, H., and Hiramoto, Y. (1987). Redistribution of fluorescently labeled tubulin in the mitotic apparatus of sand dollar eggs and the effects of taxol. Cell Struct. Funct., 12: 43-52.

14. Hiramoto, Y. and NaKano, Y. (1988). Micromanipulation studies of the mitotic apparatus in sand dollar eggs. Cell Motil. Cytoskeleton, 10: 172-184. 
15. Inoute, S. (1952). The effect of colchicine on the microscopic and submicroscopic structure of the mitotic spindle. Exp. Cell Res., (Suppl.) 2: 305-318.

16. Inoué, S. (1981). Cell division and the mitotic spindle. J. Cell Biol., 91: 1315-1475.

17. Khawaja, S., Gundersen, G.G., and Bulinski, J.C. (1988). Enhanced stability of microtubules enriched in detyrosinated tubulin is not a direct function of detyrosination level. $J$. Cell Biol., 106: 141-149.

18. Kilmartin, J.V., Wright, B., and Milstein, C. (1982). Rat monoclonal anti-tubulin antibodies derived by using a new nonsecreting rat cell line. J. Cell Biol., 93: 576-582.

19. OKa, M.T., Arai, T., and Hamaguchi, Y. (1990). Heterogeneity of microtubules in dividing sea urchin eggs revealed by immunofluorescence microscopy.: Spindle microtubules are composed of tubulin isotypes different from those of astral microtubules. Cell Motil. Cytoskeleton, 16: 239-250.

20. Piperno, G., Ledizet, M., and Chang, X. (1987). Microtubules containing acetylated $\alpha$-tubulin in mammalian cells in culture. J. Cell Biol., 104: 289-302.

21. RaFf, E.C. (1984). Genetics of microtubule systems. J. Cell Biol., 99: 1-10.

22. RAPPAPORT, R. (1986). Establishment of the mechanism of cytokinesis in animal cells. Int. Rev. Cytol, 105: 245-281.

23. Rebrun, L.I. and Sawada, N. (1969). Augmentation and dispersion of the in vivo mitotic apparatus of living marine eggs. Protoplasma, 68: 1-22.

24. RIEDER, C.L. (1982). The formation, structure, and composi- tion of the mammalian kinetochore and kinetochore fiber. Int. Rev. Cytol., 79: 1-58.

25. SALMON, E.D. and BEGG, D.A. (1980). Functional implications of cold stable microtubules in kinetochore fibers of insect spermatocytes during anaphase. J. Cell Biol., 85: 853-865.

26. Salmon, E.D., Leslie, R.J., Saxton, W.M., Karow, M.L., and Mcintosh, J.R. (1984). Spindle microtubules dynamic in sea urchin embryos.: Analysis using a fluorescein-labeled tubulin and measurements of fluorescence redistribution after laser photobleaching. J. Cell Biol., 99: 2165-2174.

27. Salmon, E.D., Mckeel, M., and Hays, T. (1984). Rapid rate of tubulin dissociation from microtubules in the mitotic spindle in vivo measured by blocking polymerization with colchicine. $J$. Cell Biol., 99: 1066-1075.

28. SCarcello, L.A., Janicke, M.A., and LaFountain, J.R., Jr. (1986). Kinetochore microtubules in crane-fly spermatocytes.: Untreated, $2^{\circ} \mathrm{C}$-treated, and $6{ }^{\circ} \mathrm{C}$-grown spindles. Cell Motil. Cytoskeleton, 6: 428-438.

29. SLUDER, G. (1976). Experimental manipulation of the amount of tubulin available for assembly into the spindle of dividing sea urchin eggs. J. Cell Biol., 70: 75-85.

30. SNyder, J.A., Vogt, S.I., and Mclelland, S.L. (1983). Nocodazole pretreatment in anaphase selectively reduces anaphase B in PtK1 cells. Cell Motil., 3: 79-91.

31. SNyder, J.A., Golub, R.J., and BerG, S.P. (1985). Role of non-kinetochore microtubules in spindle elongation in mitotic PtK1 cells. Eur. J. Cell Biol., 39: 373-379.

(Received for publication, November 27, 1990 and in revised form, January 31, 1991) 